\title{
The natural course of inflammatory bowel disease-indeterminate from childhood to adulthood: within a 25 year period
}

This article was published in the following Dove Press journal:

Clinical and Experimental Gastroenterology

22 July 2013

Number of times this article has been viewed

\author{
Hoda M Malaty' \\ Seema Mehta ${ }^{2,3}$ \\ Bincy Abraham' \\ Elizabeth A Garnett ${ }^{4}$ \\ George D Ferry ${ }^{2,3}$ \\ 'Department of Medicine, \\ ${ }^{2}$ Department of Pediatrics, ${ }^{3}$ Texas \\ Children's Hospital, Baylor College \\ of Medicine, Houston, Texas, USA; \\ ${ }^{4}$ University of California, San \\ Francisco, California, USA
}

Background: Inflammatory bowel disease (IBD)-indeterminate is a subgroup of IBD that has features of both ulcerative colitis (UC) and Crohn's disease (CD).

Aims: To determine the clinical course of IBD-indeterminate in children over a 25 year period.

Methods: We performed a retrospective investigation on children diagnosed with IBD. Diagnosis and disease distribution of IBD was based on clinical, radiologic, endoscopic, and histologic examinations.

Results: Four hundred and twenty children diagnosed with IBD between 1986 and 2003 were identified from the IBD registry, 78 (22\%) of whom were diagnosed with IBD-indeterminate. The mean age at diagnosis was $9.2 \pm 4$ years and the mean follow-up period was $4.1 \pm 2$ years. In 2003, 18 of 78 children (23\%) were reclassified by the same physician based on the endoscopic and pathologic findings as follows: eight children with $\mathrm{CD}$, five children with $\mathrm{UC}$, and five children with non-IBD (eg, eosinophilic colitis). During 2011, 20 of the 60 patients who had maintained an IBD-indeterminate diagnosis were located and contacted, and detailed telephone interviews were conducted by the corresponding author. Two patients were reclassified as having CD (10\%), one patient was reclassified as having eosinophilic colitis (5\%), six patients remained with IBD-indeterminate $(30 \%)$, and eleven patients $(55 \%)$ reported a complete resolution of their symptoms. The follow-up period ranged from 10-18 years (mean $12.5 \pm 3$ years). Children who were reclassified as having $C D$ were significantly younger than those who maintained an IBD-indeterminate diagnosis (6.4 \pm 4 years versus $11.2 \pm 3$ years, respectively, $P=0.05)$.

Conclusion: Children with IBD-indeterminate remain classified as IBD-indeterminate, or were clinically reclassified as CD or non-IBD, or became asymptomatic as they transitioned into adulthood. The need for IBD-indeterminate classification is of importance, especially when deciding on management and treatment.

Keywords: IBD-indeterminate, children, epidemiology, clinical course

\section{Introduction}

Inflammatory bowel disease (IBD) refers to a group of diseases of which Crohn's disease (CD) and ulcerative colitis (UC) are the two main types. An estimated $20 \%$ to $30 \%$ of patients with IBD have the onset of symptoms before the age of 18 years. ${ }^{1-5}$ IBDindeterminate is a subgroup of IBD that has features of both UC and CD. Despite the several classification systems, and clinical, endoscopic, radiologic, and histopathologic tools, differentiation between IBD-indeterminate from CD and UC is still difficult. ${ }^{6,7} \mathrm{~A}$ large population based study has reported that patients with IBD-indeterminate had a significantly younger age (mean \pm standard error of the mean; $9.53 \pm 4.8$ years) at diagnosis when compared with patients with $\mathrm{CD}(12.4 \pm 3.8$ years; $P<0.001)$ but not when 
compared with patients with UC $(7.41 \pm 3.5$ years $) .{ }^{6}$ The overall prevalence of IBD-indeterminate ranges from 3\%-29\%. ${ }^{3-6}$ This figure has not changed over the past 30 years despite the introduction of newer diagnostic modalities. ${ }^{1,2,5-8}$

Because of the widespread and growing use of endoscopy and biopsy, the diagnosis of IBD-indeterminate is, in most cases, based on microscopic and clinical features when no definitive diagnostic features of either CD or UC can be found. The continued presence of IBD-indeterminate diagnoses supports the concept that IBD represents a spectrum of diseases rather than just two entities, CD and UC, and over time, the concept of IBD-indeterminate has evolved.

IBD-indeterminate is more frequently found in children than in adults. ${ }^{9-11}$ However, it remains unclear whether IBD-indeterminate constitutes a problem of terminology, classification, or possibly an early stage of IBD distinct from $\mathrm{CD}$ and UC. Because comprehensive population based studies are lacking, we aimed to study the clinical course of IBD-indeterminate and examine the distribution, frequency, clinical presentation, and characteristics of IBDindeterminate among a cohort of children diagnosed with IBD over a 25 year period.

\section{Materials and methods Patient population}

A retrospective epidemiologic investigation was conducted to identify a cohort of children diagnosed with IBD and who were registered in the IBD Center at Texas Children's Hospital (TCH). The hospital is located in the greater Houston metropolitan area which consists of ten counties with a diverse racial and ethnic population of 5.5 million. ${ }^{12,13}$ Our study included children with a confirmed diagnosis of IBD that was based on clinical, radiological, endoscopic, and histological criteria. ${ }^{14,15}$ The diagnosis of UC, CD, and IBDindeterminate were classified after endoscopy/colonoscopy using histological criteria. This classification was performed after the initial visit and after each follow-up visit. The cohort of children with IBD-indeterminate was entered into the IBD Registry and diagnosed in the period from 1986 to 2003.

The Pediatric IBD Registry includes data of all children diagnosed with IBD and contains a manual of procedure with all the coded study variables. The registry was in compliance with the Health Insurance Portability and Accountability Act. ${ }^{16}$ The IBD registry obtained institutional review board approval for the protocol at the Baylor College of Medicine. Informed consent and assent were obtained from parents and patients before entry of the patient's information into the registry.

\section{Data collection}

Demographic data obtained included month and year of birth, gender, and race/ethnic background. Initial IBD diagnosis was determined as CD, UC, or IBD-indeterminate. Anthropometric data (height and weight) were documented for each visit and corresponding body mass indices (BMI) were determined. BMI/age and height/age Z-scores were calculated. Medications reviewed included the following: azulfidine (sulfasalazine) and other 5-aminosalicylic acids ([5-ASAs] oral, enema, or suppository); steroids (IV and oral prednisone or prednisolone); antibiotics (such as Flagyl [metronidazole]); immunomodulators (azathioprine, 6-mercaptopurine, methotrexate, and cyclosporine); and infliximab. Patients were classified as having been exposed to medication if it was started at any time from diagnosis or during the follow-up period.

\section{Outcome measures}

The occurrence of extraintestinal manifestations (EIMs) was documented at the onset of diagnosis and at the end of the study period. EIMs were defined as arthritis, aphthous stomatitis, arthralgia, erythema nodosum, skin lesions, and/or primary sclerosing cholangitis. Osteoporosis/osteopenia was counted as a disease outcome for IBD-indeterminate. Hospitalization and surgery during the time frame of the study were also considered as outcome measures.

\section{Statistical analysis}

The baseline study was between 1986 and 2003 to increase the sample size for meaningful analysis. Each patient was counted once at entry and must have had at least one follow-up visit from the day of enrollment to the end point of the study. Age was identified based on the date of the first diagnosis of the IBD subtype. The $95 \%$ confidence intervals were calculated. Paired and unpaired $t$-tests and $\chi^{2}$ tests were used to examine the effect of age, sex, and race/ethnicity on the rate of change of IBD-indeterminate. Because the age distribution was skewed, the Kolmogorov-Smirnov test was applied to compare the age of diagnosis.

\section{Results}

Population characteristics of children with IBD-indeterminate at baseline (1986 to 2003)

Four hundred and twenty children with first diagnosis of IBD were identified from the IBD registry between 1986 and 2003. Among these patients, 78 (22\%) were classified as having IBD-indeterminate at the time of diagnosis. The mean 
age at diagnosis of children with IBD-indeterminate was significantly younger than children with either $\mathrm{CD}$ or UC $(9.2 \pm 4$ years versus $11.7 \pm 3$ years and $11.2 \pm 4$ years, respectively, $P=0.04)$. There were $64(82 \%)$ Caucasians, six (8\%) Hispanics, six (8\%) African Americans, and two (2\%) were Asian Pacific Islanders. There were 42 females.

\section{The changing course of diagnosis of IBD-indeterminate (I986 to 20 I I)}

By 2003, of the 78 children diagnosed with IBD-indeterminate, 60 children (77\%) remained diagnosed with IBDindeterminate after a mean follow-up time of $3.5 \pm 2.7$ years and 18 children (23\%) were reclassified as follows: eight children with $\mathrm{CD}$, five children with $\mathrm{UC}$, and five children as non-IBD (eg, an eosinophilic colitis diagnosis was based on histological examination) after a mean follow-up period of $4.1 \pm 2$ years (Figure 1).

During 2011, we were able to locate 20 of the 60 patients who had maintained an IBD-indeterminate diagnosis and detailed telephone interviews were conducted. After the detailed interview, and based on the information from the patients and their physicians, the corresponding author was able to perform the following reclassification: two patients with $\mathrm{CD}(10 \%)$, one patient as eosinophilic colitis (5\%), six patients remained with IBD-indeterminate $(30 \%)$, and eleven patients (55\%) reported a complete resolution of their symptoms and did not receive any medications after their last visit. The follow-up period ranged from 10-18 years (mean $12.5 \pm 3$ years). Children who were reclassified as $\mathrm{CD}$ were younger than those in whom the diagnosis did not change (6.4 \pm 4 years versus $11.2 \pm 3$ years, respectively, $P=0.05$ ) (Figure 1).

We compared the demographic variables between the 20 patients we contacted to the other 40 patients. No significant difference was found between both groups with respect to sex (55\% female versus $45 \%$ male, $P=0.08$ ) or race/ethnicity ( $90 \%$ Caucasian versus $10 \%$ non-Caucasian, $P=0.8)$. Moreover, there was no difference in the mean age at diagnosis $(8.4 \pm 4.7$ years versus $10.1 \pm 4.2$ years, $P=0.07)$.

\section{EIMs of patients with IBD-indeterminate (1986 to 2003)}

At entry, only five patients (6\%) experienced one or more EIMs of whom three patients $(60 \%)$ were diagnosed with peripheral arthritis, one patient (20\%) had erythema nodosum, and one

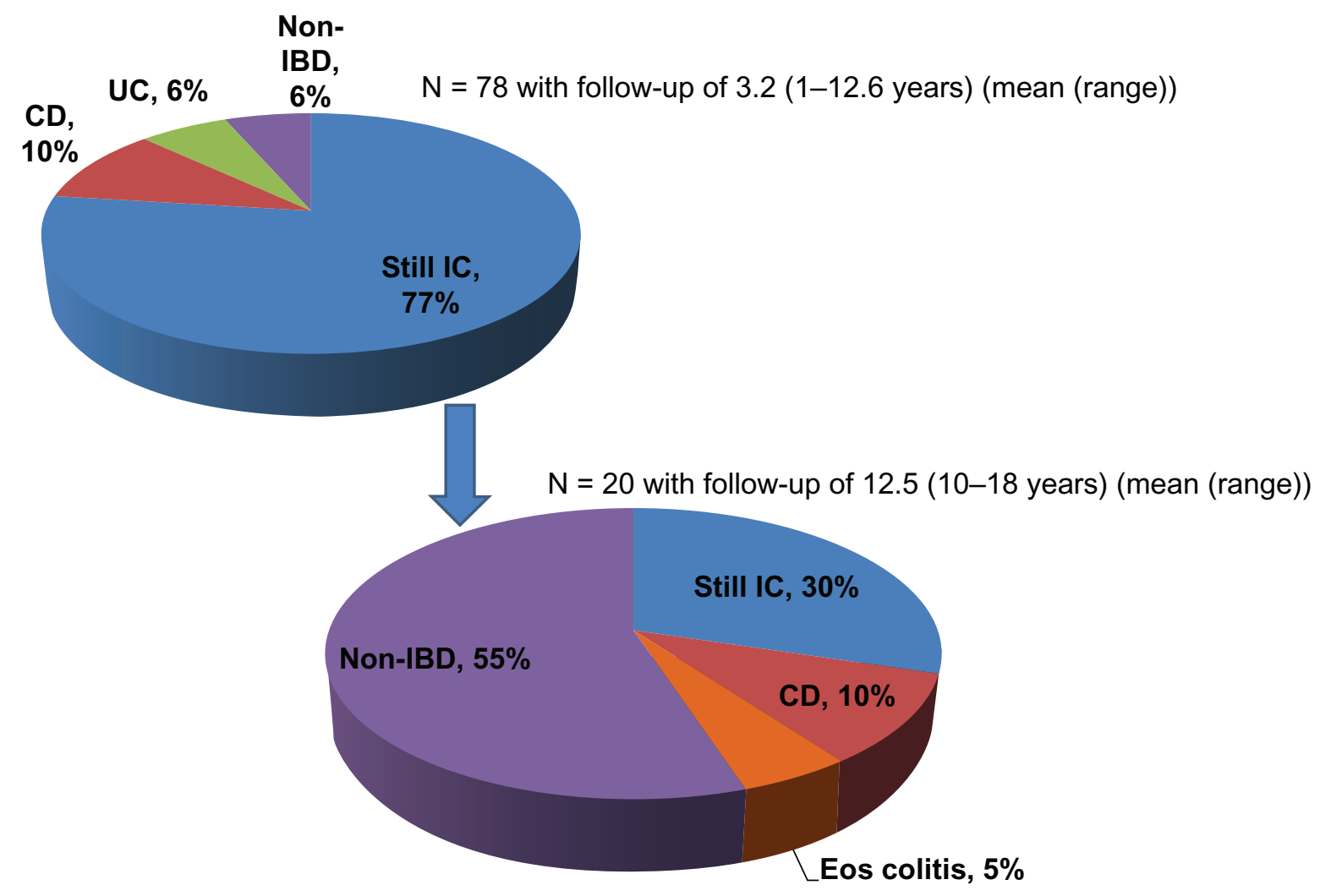

Figure I The changing course of diagnosis in 201 I of the 60 children who maintained indeterminate colitis diagnosis in 2003.

Abbreviations: CD, Crohn's disease; IBD, inflammatory bowel disease; IC, indeterminate colitis; UC, ulcerative colitis; Eos, eosinophilic. 
patient had apthous stomatitis (20\%). Only one patient had two EIMs: peripheral arthritis and sclerosing cholangitis. Four of the five patients developed EIMs after confirmed diagnosis of IBD-indeterminate and only one patient had peripheral arthritis before the IBD-indeterminate diagnosis was confirmed.

Because of the controversy of inclusion of osteopenia/ osteoporosis as EIMs, it was excluded at the study entry and was considered as a postdiagnostic EIM. There was one child diagnosed with osteoporosis/osteopenia after the IBDindeterminate diagnosis. EIMs were not associated with age, sex, or ethnicity. However, children with EIMs had a longer follow-up duration than those without EIMs (5.2 \pm 2.6 years versus $3.1 \pm 2.2$ years)

Seven IBD-indeterminate children were hospitalized during the study period, and two patients were reclassified as CD and one patient as UC by the end of the study. None of the children underwent colectomy.

\section{Growth (1 986 to 2003)}

We characterized the growth pattern of children with IBDindeterminate at both their initial presentation and at maximal follow-up. At the initial visit, $5 \%$ of the children ( 4 of 78 children) had a height Z-score of less than -2sd (less than 2 standard deviations below normal) and by the final visit, 9\% (7 of 78 children) had a height Z-score of less than -2 sd. The overall difference in height $\mathrm{Z}$-score did not improve for all patients from the initial to final visit $(-0.34 \pm 0.97$ versus $-0.42 \pm 0.99$, $P=0.11$ ). IBD-indeterminate patients did not demonstrate a difference in BMI from the initial to the final visit.

\section{Laboratory results and symptoms profile (1986 to 2003)}

At entry, the albumin measures ranged between 3 and $5 \mathrm{~g} / \mathrm{dl}$ with an average of $4.4 \mathrm{~g} / \mathrm{dl}$, hematocrit values ranges from
$30 \%$ to $46 \%$ with an average of $37.5 \%$, and the average erythrocyte sedimentation rate (ESR) was 11.8 , and there were eight patients who had ESR over 20 of whom five $(62.5 \%)$ were among those who were reclassified as CD.

For the stool pattern at diagnosis, $11 \%$ of patients had gross bleeding of $\geq 6$ stools that were liquid or nocturnal diarrhea, 49\% had 1-2 semiformed stool with blood or 2-5 liquid stool, and $40 \%$ of patients were normal.

Seventy five percent of patients had no tenderness or mass during abdominal examination at entry, 23\% had tenderness and/or mass, and only $2 \%$ had tenderness with involuntary guarding and/or definite mass. Because the data from this current study started in 1986, the physician global assessment was available for only 29 patients at their first clinic visits. Two patients reported frequent limitation of activity, eight patients reported occasional difficulty and 19 reported no limitations.

\section{Medical treatment received at initial and at maximal follow-up (1986 to 2003)}

At diagnosis, $46 \%$ of the IBD-indeterminate patients were treated with oral 5-ASAs, 36\% received oral steroids, and 7\% received immunomodulators (eg, 6-mercaptopurine/Imuran) (Figure 2). The majority of patients who received systemic steroids were still using them at, and after, 4 years from diagnosis. Of the 51 subjects on steroids during the study period, $17(33 \%)$ also received immunomodulators. However, only one of the 18 subjects on immunomodulators did not also take steroids during the follow-up period (Figure 2).

Rectal 5-ASAs were being prescribed at diagnosis to one patient and this increased to three patients by the end of the first year suggesting that these patients continued to have distal disease. None of the patients were prescribed infliximab at diagnosis. Only one patient received infliximab

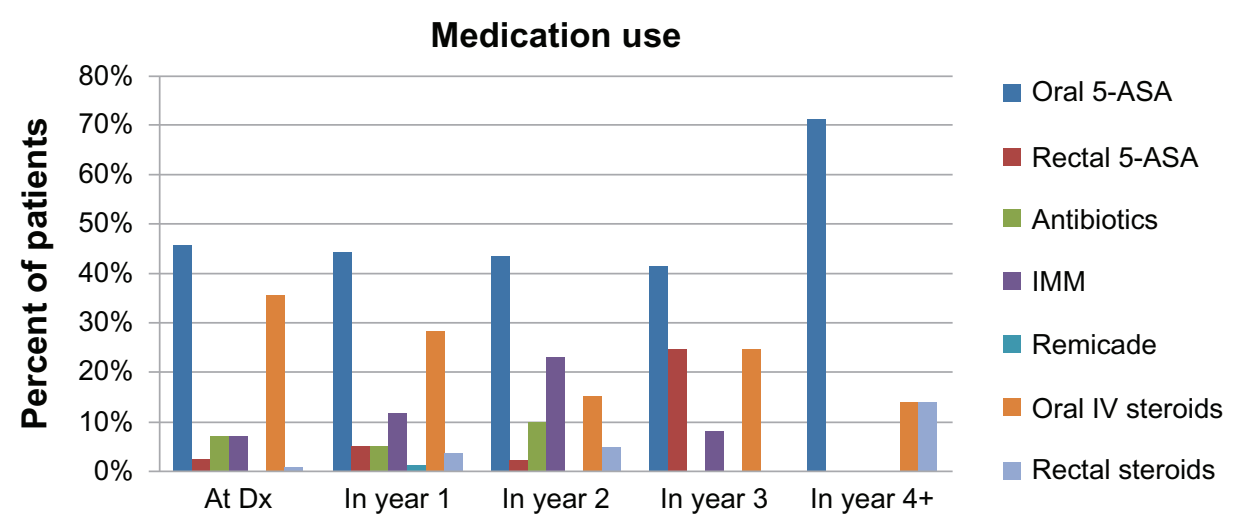

Figure 2 Treatments received at maximal follow-up in 78 pediatric patients with indeterminate colitis. Abbreviations: 5-ASA, 5-aminosalicylic acids; Dx, diagnosis; IMM, immunomodulator; IV, intravenous. 


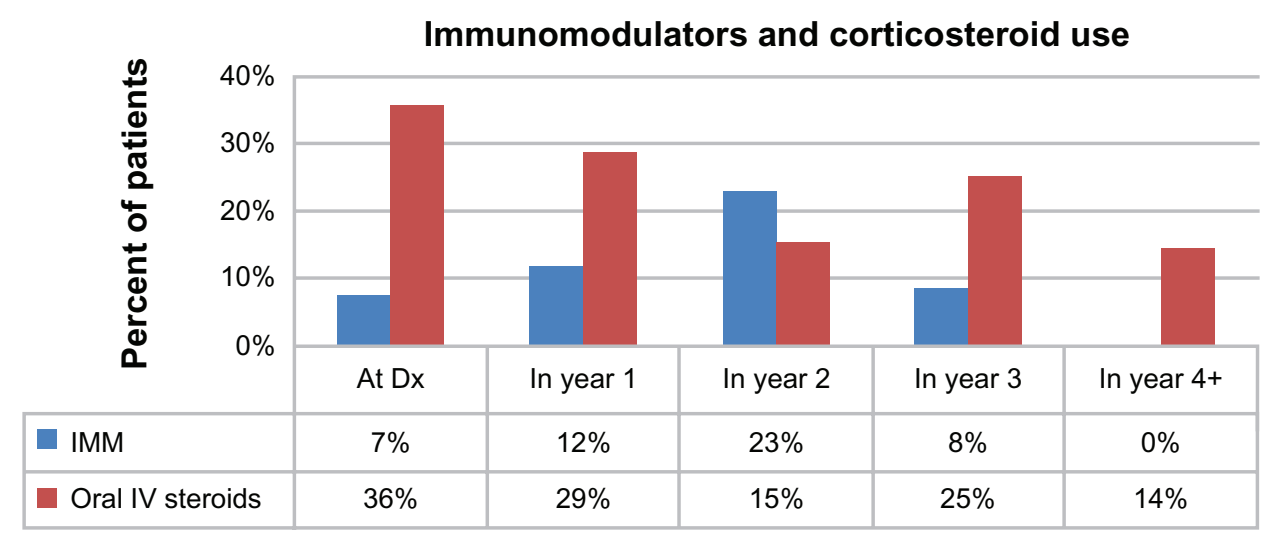

Figure 3 Steroid treatment received by $5 \mathrm{I}$ pediatric patients with indeterminate colitis who received immunomodulators at maximal follow-up. Abbreviations: Dx, diagnosis; IMM, immunomodulator; IV, intravenous.

starting in 1998 as the drug was approved for use in the US in 1998.

\section{Discussion}

The main strengths of our study emerged from its design and the availability of the pediatric IBD Registry with its detailed medical records as there is a lack of such studies in pediatric patients. Moreover, the diagnosis of the three subtypes of IBD was confirmed as all patients had a complete colonoscopy and histological evaluation at diagnosis and during the follow-up period. All patients included in the analysis had a follow-up duration of at least 2 years.

Our findings have shown that IBD-indeterminate has a distinctive clinical pattern of IBD in children with a higher prevalence than that reported in adults. ${ }^{9,10}$ Only a small number of the studied IBD-indeterminate children were reclassified to either $\mathrm{CD}$ or $\mathrm{UC}$, similar to what has been reported by Lindberg and coworkers ${ }^{11}$ in a Swedish based population study of children with indeterminate colitis (IC). In that study, only 29 of 171 patients with IBD-indeterminate (previously called IC) were ultimately reclassified as either CD or UC. Of interest, the majority of the original IC patients ended up with the same IC diagnosis or with a non-IBD diagnosis. There is still controversy on the reclassification of IBD among adults and children and there are discordant results in different populations. While a study from northern Italy has reported that $80 \%$ of patients initially diagnosed with IC declared themselves with either CD or UC after a median follow-up period of 8 years, ${ }^{8}$ a study from Finland found that in the long-term natural course of surgically treated IC patients after a median 5.5 years of follow-up, clinicopathology confirmed typical CD in $50 \%$ of the cases, and the final diagnosis remained IC in the other $50 \%$ of the cases. $^{17}$
Only $6 \%$ of children in this study had one or more EIMs at diagnosis which is a much lower rate than what is usually reported among CD and UC patients $(25 \%-30 \%) .{ }^{18-20}$ Moreover, none of the children in this study had colectomy. These findings support the high rate of reclassification of IBD-indeterminate to non-IBD in our study. Up to $70 \%$ of the cohort were maintained by receiving 5-ASAs by the fourth year of follow-up, which is in accordance with the recent guidelines from the US and Europe that recommend 5-ASAs as first line therapy in the induction of response and remission, as well as in maintenance. ${ }^{21,22}$ To date, it is not entirely clear how well IBD-indeterminate patients respond clinically to some of the IBD therapies because these patients tend to be excluded from clinical studies.

Laboratory findings or patient symptoms at entry did not predict the changing pattern of IBD-indeterminate over time. These findings agree with a report by Thakkar et al who concluded that neither blood work nor patient symptoms before an endoscopy procedure could predict management outcome of IBD in children, emphasizing the importance of endoscopic examination. ${ }^{23}$ However, more than $60 \%$ of the patients with a very high level of ESR were reclassified as CD by the end of this study. Such findings must be taken with caution as the elevation of ESR can be a marker for any type of inflammatory process.

The utilization of data for constructing a retrospective cohort has some shortcomings. Our studied population presented to a tertiary care center from a single center, and it was possible that more severe cases were referred to an academic center. However, $\mathrm{TCH}$ is one of the largest children's hospitals in the nation offering a diverse population that closely reflects that of the Texan population. Moreover, there are only two private pediatric gastroenterology practices that opened in the same area, but not until 2000. 
The second limitation of the study is that the information on EIMs at diagnosis of IBD was retrospective and there were no standard criteria for the definition of each EIM. However, our definition for EIMs was based on what is reported in the literature. Another limitation of the study is that we were only able to locate 20 patients to follow up. Given that Houston, Texas, is a mobile society, it was a difficult and costly job to locate these patients after a long period of time that reached up to 20 years. Moreover, when we compared the demographic variables between the 20 patients we contacted to the other 40 patients, no significant difference was found between both groups. Finally, the missing information on disease location could have provided some insight as to how useful these markers are at diagnosis.

In conclusion, IBD-indeterminate is a relatively common subgroup of IBD among pediatric patients with a higher prevalence than observed in adults. Children with IBDindeterminate remained classified as IBD-indeterminate, were reclassified as non-IBD, or became asymptomatic as they transitioned into adulthood. Therefore, the need of IBDindeterminate classification is of importance, especially when deciding on management and treatment.

\section{Acknowledgment}

This work is supported by a grant from Janssen Biotech. The authors have no financial relationships relevant to this article to disclose.

\section{Disclosure}

The authors declare no conflicts of interest in this work.

\section{References}

1. Malaty HM, Fan X, Opekun AR, Thibodeaux C, Ferry GD. Rising incidence of inflammatory bowel disease among children: a 12 year study. J Pediatr Gastroenterol Nutr. 2010;50:27-31.

2. Kugathasan S, Judd RH, Hoffmann RG, et al; Wisconsin Pediatric Inflammatory Bowel Disease Alliance. Epidemiologic and clinical characteristics of children with newly diagnosed inflammatory bowel disease in Wisconsin: a statewide population-based study. $J$ Pediatr. 2003;143:525-531.

3. Loftus EV Jr. Clinical epidemiology of inflammatory bowel disease: Incidence, prevalence, and environmental influences. Gastroenterology. 2004;126:1504-1517.

4. Turunen P, Kolho KL, Auvinen A, Iltanen S, Huhtala H, Ashorn M. Incidence of Inflammatory Bowel Disease in Finnish Children, 19872003. Inflamm Bowel Dis. 2006;12:677-683.

5. Abraham B, Mehta S, El-Serag HB. Natural history of pediatric onset inflammatory bowel disease: a systematic review. J Clin Gastroenterol. 2012;46:581-590.
6. Carvalho RS, Abadom V, Dilworth HP, Thompson R, Oliva-Hemker M, Cuffari C. Indeterminate colitis: a significant subgroup of pediatric IBD. Inflamm Bowel Dis. 2006;12:258-262.

7. Burakoff R. Indeterminate colitis: clinical spectrum of disease. J Clin Gastroenterol. 2004;38:S41-S43.

8. Meucci G, Bortoli A, Riccioli FA, et al. Frequency and clinical evolution of indeterminate colitis: a retrospective multicenter study in Northern Italy. GSMII (Gruppo di Studio per le Malattie Infiammatorie Intestinali). Eur J Gastroenterol Hepatol. 1999;11:909-913.

9. Hildebrand H, Fredrikzon B, Holmquist L, Kristiansson B, Lindquist B. Chronic inflammatory bowel disease in children and adolescents in Sweden. J Pediatr Gastroenterol Nutr. 1991;13:293-297.

10. Hildebrand H, Brydolf M, Holmquist L, Krantz I, Kristiansson B. Incidence and prevalence of inflammatory bowel disease in children in south-western Sweden. Acta Pediatr. 1994;83:640-645.

11. Lindberg E, Lindquist B, Holmquist L, Hildebrand H. Inflammatory bowel disease in children and adolescents in Sweden: 1984-1995. J Pediatr Gastroenterol Nutr. 2000;30:259-264.

12. RacelEthnicity: 1980-2010 City of Houston. 1980-2000, 2010 PL94-171 Data, US Census Bureau. Available from: http://www.houstontx.gov/ planning/Demographics/docs_pdfs/Cy/coh_race_ethn_1980-2010.pdf. Accessed on June 18, 2013.

13. The City of Houston [hompage on the Internet] Demographic Data. Available from: http://www.houstontx.gov/planning/Demographics/ demog_links.html. Accessed June 18, 2013.

14. Kim SC, Ferry GD. Inflammatory bowel diseases in pediatric and adolescent patients: clinical, therapeutic, and psychosocial considerations. Gastroenterology. 2004;126:1550-1560.

15. Hyams J, Markowitz J, Otley A, et al; Pediatric Inflammatory Bowel Disease Collaborative Research Group. Evaluation of the pediatric crohn disease activity index: a prospective multicenter experience. $J$ Pediatr Gastroenterol Nutr. 2005;41:416-421.

16. US Department of Health \& Human Services [homepage on the Internet]. Health Information Privacy: The Health Insurance Portability and Accountability Act of 1996 (HIPAA) Privacy, Security and Breach Notification Rules. Available from: http://www.hhs.gov/ocr/privacy/. Accessed June 18, 2013.

17. Kangas E, Matikainen M, Mattila J. Is "indeterminate colitis" Crohn's disease in the long-term follow-up? Int Surg. 1994;79:120-123.

18. Eidelwein AP, Thompson R, Fiorino K, Abadom V, Oliva-Hemker M. Disease presentation and clinical course in black and white children with inflammatory bowel disease. J Pediatr Gastroenterol Nutr. 2007;44: $555-560$.

19. White JM, O'Connor S, Winter HS, et al. Inflammatory bowel disease in African American children compared with other racial/ethnic groups in a multicenter registry. Clin Gastroenterol Hepatol. 2008;6:1361-1369.

20. Jose FA, Garnett EA, Vittinghoff E, et al. Development of extraintestinal manifestations in pediatric patients with inflammatory bowel disease. Inflamm Bowel Dis. 2009;15:63-68.

21. Ferry GD, Kirschner BS, Grand RJ, et al. Olsalazine versus sulfasalazine in mild to moderate childhood ulcerative colitis: results of the Paediatric Gastroenterology Collaborative Research Group Clinical Trial. J Pediatr Gastroenterol Nutr. 1993;17:32-38.

22. Baumgart DC, Sandborn WJ. Inflammatory bowel disease: clinical aspects and established and evolving therapies. Lancet. 2007;369: 1641-1657.

23. Thakkar K, Lucia CJ, Ferry GD, et al. Repeat endoscopy affects patient management in pediatric inflammatory bowel disease. Am J Gastroenterol. 2009;104:722-727. 
Clinical and Experimental Gastroenterology

Dovepress

\section{Publish your work in this journal}

Clinical and Experimental Gastroenterology is an international, peerreviewed, open access journal, publishing all aspects of gastroenterology in the clinic and laboratory, including: Pathology, pathophysiology of gastrointestinal disease; Investigation and treatment of gastointestinal disease; Pharmacology of drugs used in the alimentary tract;

Immunology/genetics/genomics related to gastrointestinal disease. This journal is indexed on CAS. The manuscript management system is completely online and includes a very quick and fair peer-review system. Visit http://www.dovepress.com/testimonials.php to read real quotes from published authors.

Submit your manuscript here: http://www.dovepress.com/clinical-and-experimental-gastroenterology-journal 